Part of Journal of Research of the National Bureau of Standards, Volume 26, June 1941

\title{
EVALUATING THE WEARING QUALITY OF CURRENCY PAPER
}

\author{
By Frederick T. Carson and Vernon Worthington
}

\section{ABSTRACT}

This report describes means which have been developed to determine the relative serviceability of currency-type papers. A reproducible crumpling and smoothing cycle has been developed with which to imitate the appearance and characteristics of worn paper currency. The changes in significant physical properties of this worn paper are then measured. An automatic, motor-driven crumpling apparatus is used, which alternately crumples and straightens out a specimen at a rate of about seven times per minute. The change in air permeability as the crumpling procedure progresses appears to be the most useful single measure thus far found of the decreasing serviceability of the paper. An air permeameter, specially designed for use in conjunction with the crumpling apparatus, is briefly described.

\section{CONTENTS}

I. Introduction

II. Crumpling cycle

III. Crumpling apparatus... 469

IV. Measuring the effect of crumpling

1. Strength _...

2. Stiffness........ 473

3. Air permeability

(a) Value of air-permeability measurements .......... 475

V. Conclusion (b) Description of the air-permeability instrument...... 477

\section{INTRODUCTION}

For several years the National Bureau of Standards has been engaged in research, supported by the Bureau of Engraving and Printing, to find means of increasing the useful life of the United States paper currency. Replacement of worn-out paper currency requires an annual expenditure of several million dollars. Any improvement leading to a longer average life, therefore, represents a substantial saving.

Changes made in composition, fabrication, and processing of experimental papers, in order to improve the serviceability of paper currency, have sometimes been appraised by the United States Treasury Department by means of actual service tests. Such tests are much too slow, however, for routine evaluations, and may be inconclusive, owing to the difficulty of getting enough returns on comparable treatments in service. A standardized laboratory test, capable of simulating in a comparatively short time the type of wear 
which paper currency undergoes in circulation, is essential in this effort to further improve the serviceability of currency paper.

Wear of paper currency differs from wear in its more common connotation, inasmuch as abrasion, loss of substance, and breaking are not common causes of failure. Repeated folding and crumpling, and the incident soiling and defacing, are the primary factors responsible for withdrawal of worn United States paper currency from circulation.

Various laboratory methods of wearing the paper have been tried, such as the conventional folding and bending tests, the rotating-drum tumbling apparatus, and several specially devised methods of twisting, rubbing, and bending the paper. Of the various types of treatment used, crumpling the paper seems best to simulate the type of wear which the notes undergo in circulation. It also wears the test specimen fairly uniformly over its entire surface, and has in this respect a distinct advantage over the other wear tests that were studied. The creasing pattern and general appearance of worn-out paper currency are fairly well simulated by the random creasing which occurs in the crumpling operation.

The paper which has been used in this work is a special issue of nondistinctive, currency-type paper, similar to the regular issue, except that the distinctive, colored fibers have been left out. The paper was printed in the usual manner with a specially engraved plate.

\section{CRUMPLING CYCLE}

A definite procedure, involving mechanical aids, seems necessary in order to make the crumpling treatment reproducible. Considerable
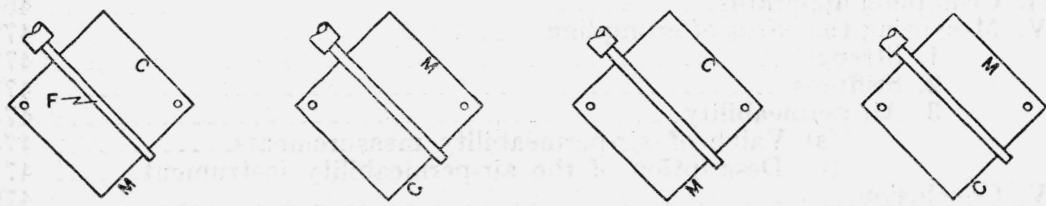

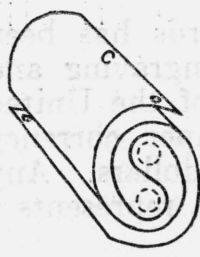

1

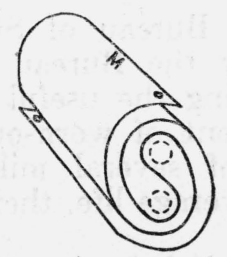

II

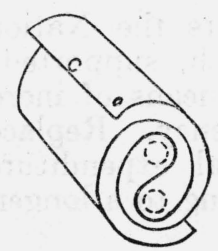

III

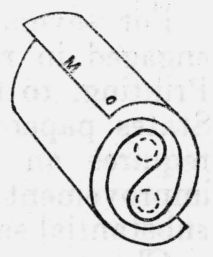

IV

FIGURE 1.-Sketch illustrating the four-phase crumpling cycle.

$C$ and $M$ designate two edges parallel respectively to the cross direction and the machine direction of the paper. I and III are crushed in the cross direction; II and IV in the machine direction. III and IV are rolled (by the fork, $F$ ) in the opposite direction from I and II. The lower sketches are much enlarged over the upper ones.

experimenting with various crumpling procedures and mechanical models resulted in the choice of a procedure and crumpling cycle wherein a square of paper is rolled up, slipped into a hollow cylinder, 
and crushed endwise of the roll to a predetermined volume by means of a piston. This crumpling procedure is repeated, with variations in a definite order, as many times as may be required. The crumpling cycle, involving four combinations of the directions of rolling and crumpling, is illustrated by figure 1 .

The specimen $2 \frac{5}{8}$ inches on a side (the largest square obtainable from the present paper currency), is rolled, S-shaped, from the center by a two-tined fork, $F$, placed astride the specimen parallel with one or the other (alternately) of the two principal directions of the paper. As a result the paper roll is crushed by the piston, one time in the cross direction and the next time in the machine direction. Furthermore, in the first two phases of the cycle the fork is rotated in one direction and in the third and fourth phases it is rotated in the opposite direction. Bending the paper thus in opposite directions helps to break up preceding patterns of wrinkles and promotes a more even distribution of the creases.

Tests were made also with the simpler two-phase cycle (I and II only of figure 1), in which the specimen is crushed alternately in the machine direction and the cross direction, but is always rolled up in the same direction. It was somewhat surprising to find that the effect produced in this way by a given number of crumplings was only about half as great as that resulting from the four-phase cycle. The latter appears to promote a more general distribution of the creases, and, although somewhat complicated, produced such good results in the early experiments that the further development of this form of test seemed amply justified.

\section{CRUMPLING APPARATUS}

The crumpling cycle shown in figure 1 is accomplished by an automatic, motor-driven crumpling apparatus, illustrated by figures 2 , 3,4 , and 5. A simpler, manually operated crumpling device was used in the earlier work, but the difficulty of preserving the necessary sequence in the various manipulations in the cycle led to the development of the automatic apparatus.

In the crumpling apparatus the specimen is secured by means of cords passed through eyelets placed in two opposite corners of the specimen, as shown in figure 2 . The square of paper is rolled up by the fork, $F$, after which it is drawn into the crumpling cylinder, $C$, and crumpled to a small wad between the gate, $G$, and the piston, $P$. In subsequent operations the wad is expelled and opened out by the tension lever, $T$, and the smoothing arms, $A 1$ and $A \mathscr{2}$, again rolled up and crumpled in the opposite direction, and so on according to the cycle illustrated in figure 1. A more detailed description of the operation and function of the various mechanisms will make the process clearer.

Figure 2 shows schematically the most important features of the apparatus. All the mechanisms that perform the various manipulations incident to the rolling, crumpling, and straightening of the specimen require intermittent motions. The mechanisms are, therefore, driven and timed by suitably shaped cams that operate on appropriate levers and linkages. The cams are mounted on a common shaft, $S 1$, which is driven at about 7 revolutions per minute by 
a motor and reducing gear (see fig. 3). The specimen is crumpled once each revolution of the camshaft.

At the beginning the fork, $F$, is in the broken-line position astride the tension cord, $H$, and out of contact with the specimen, $W$. The specimen, freely suspended under slight tension, tends to stand in a vertical plane. It is so constrained by the cord that joins the specimen to the piston-a horizontal, $V$-shaped loop, $V$, with its ends attached at the two ends of a diameter of the piston and its

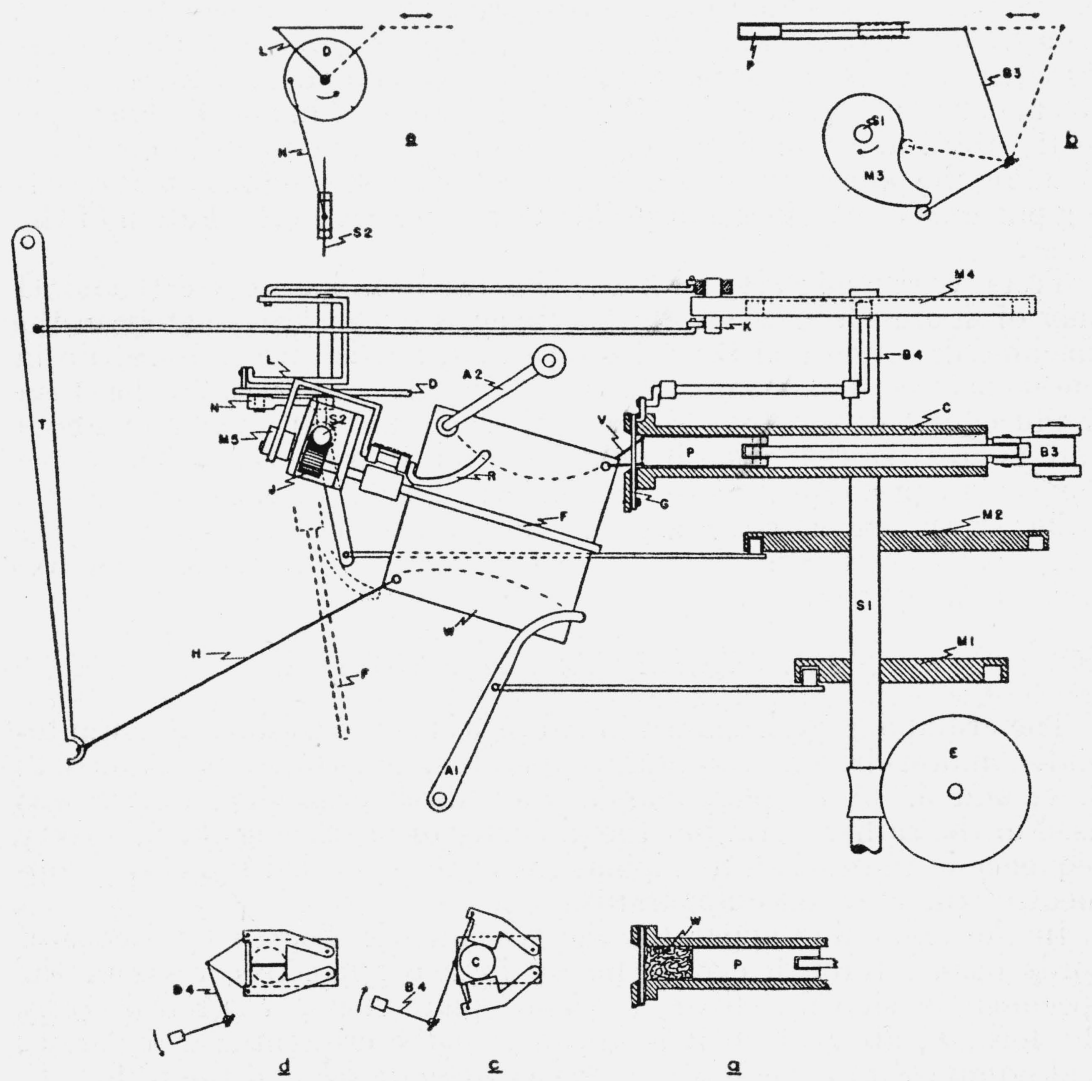

FIGURE 2.-Schematic drawing of the automatic crumpling apparatus.

apex in the eyelet. As the fork, $F$, swings about the vertical shaft, S2, as a pivot, approaching the corner of the specimen from its brokenline position, a curved finger, $R$ (see also fig. 4), moving above the cord, $H$, carries the upper half of the specimen backward, forcing it toward the horizontal position. The fork then follows across the corner, astride the specimen to the middle, as shown by the full-line position in figure 2. In this position the fork stops its swing momentarily and begins to rotate. As the specimen is rolled up, the tension is relaxed, and the fork swings the roll into line with the crumpling cylinder. Friction plates, $Z$ (figs. 4 and 5 ), flanking the fork above and below, aid in holding the specimen tightly rolled. The swing of the fork during this time is governed by a cam, $M^{2}$, acting through appropriate linkage, while its rotation is produced by 


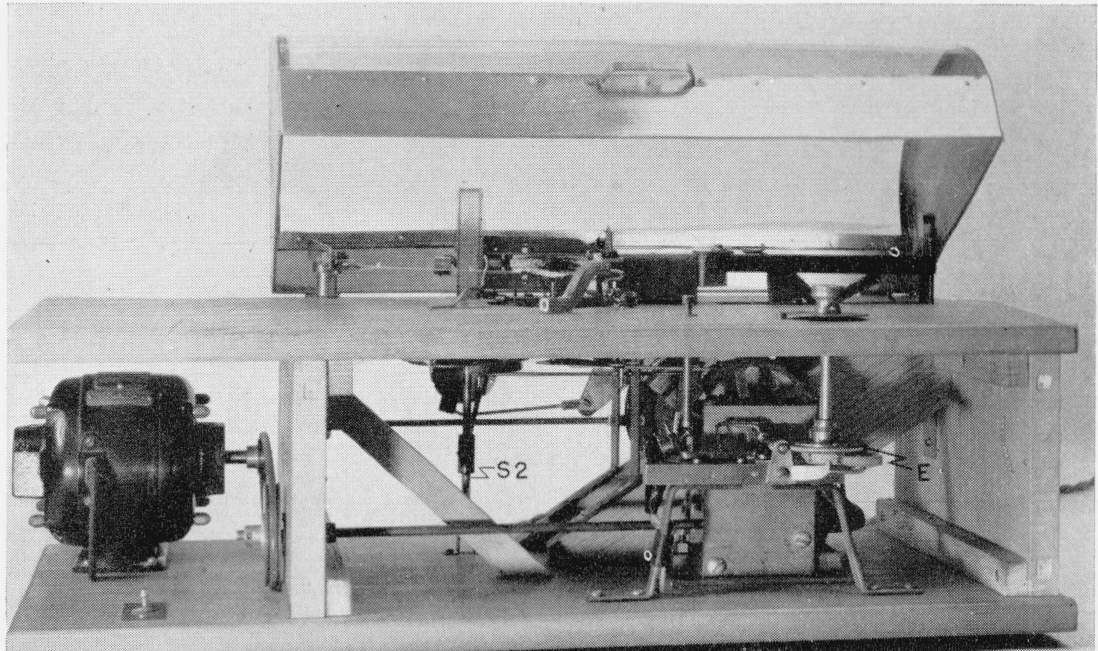

FIGURE 3.-Automatic crumpling apparatus, showing the driving mechanism.

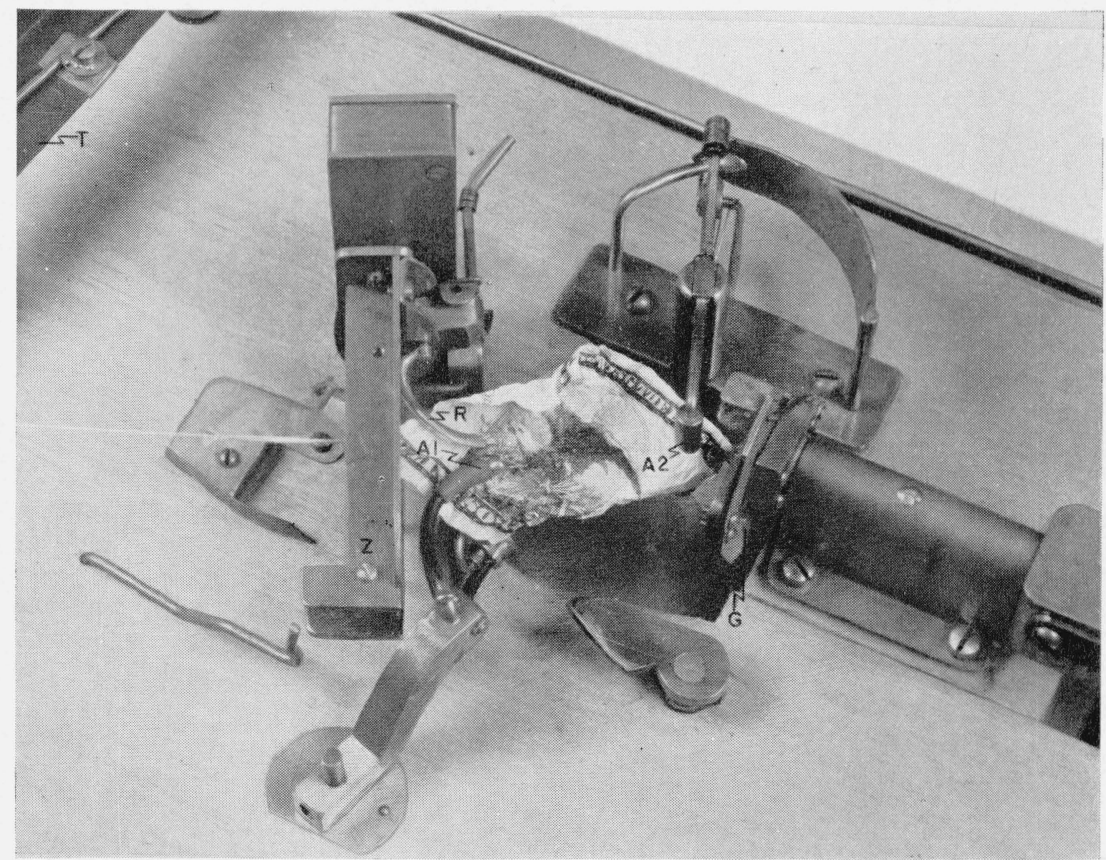

FIGURE 4.-Detail of crumpling apparatus, showing specimen being prepared for the next crumpling. 


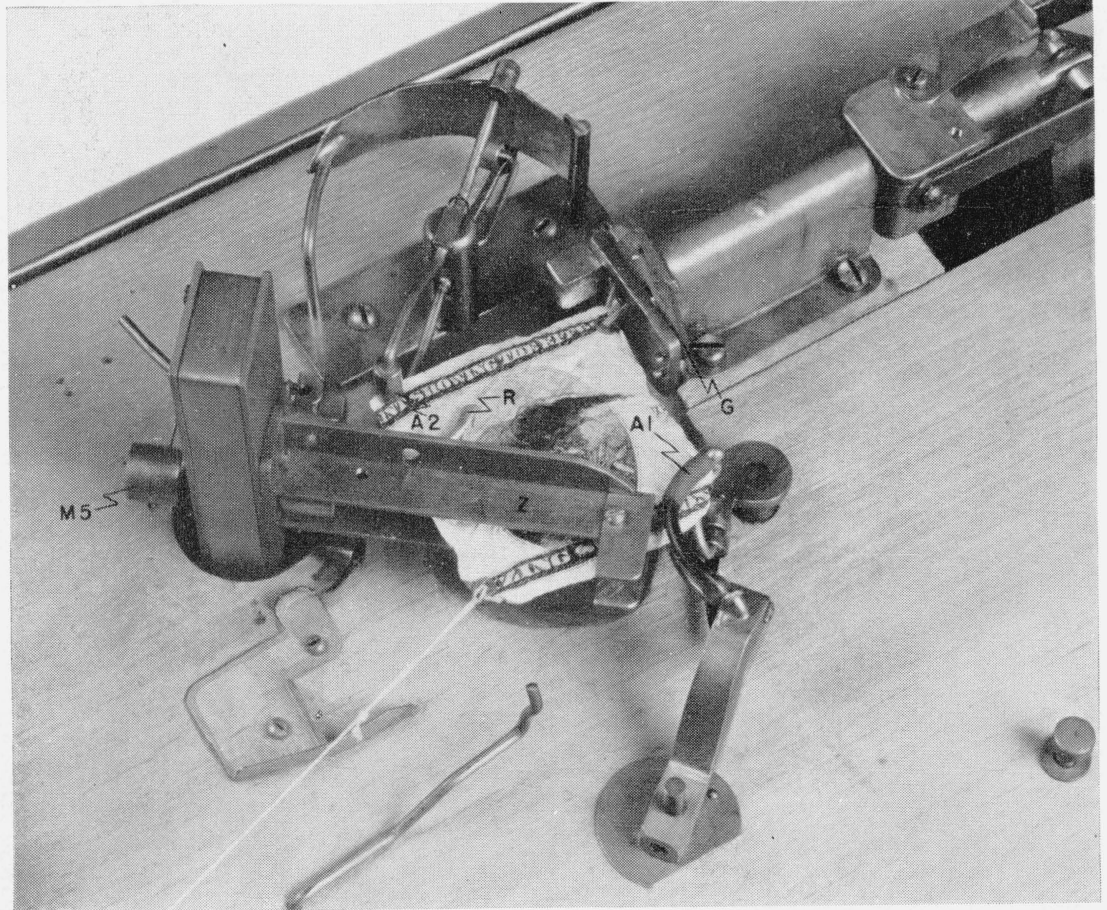

FiguRE 5.-Detail showing the specimen smoothed out ready to be rolled up and crumpled again. 
a vertically moving rack (integral with S2) which turns a pinion, $J$, on the fork shaft.

At this point the piston is retracted, pulling the rolled specimen off the fork and into the crumpling cylinder through the funnel-shaped mouth. A scissorslike gate, $G$, closes behind the roll. On the forward stroke of the piston the specimen is slowly crumpled to a small wad, as indicated in $a$ of figure 2 . In the meantime the fork has swung back to its initial broken-line position. The movement of the piston is governed by a cam, $M S$, acting on a bell-crank lever, $B 3$, as shown in $b$ (reduced scale) of figure 2, and a quick-acting return mechanism, not shown. The opening and closing of the gate, $G$, are governed by a cam, $M_{4}$, which acts through the offset bell-crank lever, $B 4$, and a toggle. The gate is shown open and closed in $c$ and $d$ of figure 2 .

After the specimen has been thus crumpled, the gate opens and the crumpled wad of paper is expelled as the piston stroke is completed. The tension lever, $T$, actuated by the crank-pin, $K$, on the cam, $M_{4}$, exerts tension and stretches the crumpled paper out along the eyeleted diagonal. As the fork swings forward the second time, the reversing finger, $R$, comes underneath the cord and therefore turns the specimen about the eyeleted diagonal in the opposite direction to that of the first time. It is obvious from figure 1 that this will reverse the relative positions of machine direction and cross direction. Whereas in the first phase of the cycle the cross direction was the axis of the rolled specimen, now the machine direction is the axis and becomes the direction of crumpling in the second phase. In this manner the apparatus alternates the direction of crumpling in successive phases of the cycle. Cam M5 on the fork shaft causes the reversing finger, $R$, to be one time above the cord and the next time below it, inasmuch as the fork makes $1 \frac{1}{2}$ revolutions each time the specimen is rolled up.

Following immediately behind the reversing finger, $R$, when it turns the specimen, as smoothing arm, $A 1$, composed of two opposed jaws slightly ajar, moves over the eyeleted corner and along an arc to the adjacent uneyeleted corner, opening out the crumpled paper as it goes. Simultaneously a somewhat similar smoothing arm, $A \mathscr{Q}$, moves from the other eyelet on an arc to straighten out the opposite edge. As the smoothing clamps approach the uneyeleted corners, they begin to close by wedge action, until finally they clamp slightly and stretch the specimen out along the uneyeleted diagonal while tension is still on the other diagonal (fig. 5). Thus the crumpled paper is flattened out and held, allowing the fork to move freely into position to roll it up again. The smoothing arm, $A 1$, is actuated by a cam, M1. The two smoothing arms are connected by a cross link (not shown), so that $A 2$ is driven by $A 1$. The design of successful smoothing arms proved to be the most obdurate problem in developing the crumpling apparatus. At some stage in the crumpling cycle a persistent fold would usually appear that the mechanism was incapable of smoothing out sufficiently to allow the fork to function normally in the next phase. Many forms of smoothing devices were tried before a successful combination was found. The smoothing arms $A 1$ and $A 2$ differ somewhat in form and operation. Figures 4 and 5 show them in different positions.

The third and fourth phases in the cycle are like the first and second, respectively (see fig. 1), except that the fork turns in the opposite direction. This reversal in direction of rotation of the fork every second phase in the cycle is accomplished by reversing the direction 
of travel of the rack and shaft, S2. This rack-and-shaft combination is moved up or down by a connecting rod, $N$, and a crank-pin attached to a disk, $D$ (see $e$ of fig. 2, and fig. 3), that makes a quarter turn for each revolution of the cam shaft, S1. Thus the rack is pushed upward during two successive phases in the cycle and downward during the next two. A ratchet device, $L$, actuated by the crank-pin, $K$, on the cam, $M_{4}$, moves the disk a quarter turn for each revolution of the cam shaft, $S 1$.

$A$ disk counter and automatic stopping device, $E$, is geared to the cam shaft, $S 1$ (see also fig. 3 ). It can be set so that it will stop the motor after any predetermined number of crumplings in multiples of 4 , from 4 to 100 , by automatically throwing a toggle switch.

Although the apparatus is by no means infallible, it performs satisfactorily, and has been used in determining the probable relative wearing quality of many samples of currency paper.

\section{MEASURING THE EFFECT OF CRUMPLING}

\section{STRENGTH}

Reproducible crumpling of the paper having been achieved, there follows the necessity of measuring the effect produced, and of measuring it in such a way as to be indicative of the probable behavior of the currency during its serviceable life. Several measurements suggest themselves as important criteria of change in significant properties as a result of the artificial wear treatment.

The change in strength of the paper is significant, although not of primary importance in the currency paper used today, inasmuch as improvements in making the paper, worked out several years ago by the National Bureau of Standards with the financial support and cooperation of the Bureau of Engraving and Printing, have resulted in a paper of such strength and durability that rupture of the paper is now not often the cause of retiring paper currency from circulation.

The easiest test of strength to make is, of course, the burstingstrength test. Unfortunately, it has not proved to be very useful, because the decrease in strength with repeated crumpling is accompanied by an increase in stretch. In the bursting-strength test these two trends offset each other, so that the bursting pressure does not change very much with the extent of the crumpling treatment.

The tensile strength does decrease considerably, and in a characteristic manner. Although it is a destructive test and cannot be used to follow the behavior of a given specimen through successive crumplings, it has been used to a considerable extent as a corroborative test of the end result of a series of crumplings. The square specimen is cut into four strips running in the crosswise direction, which are tested on a conventional paper tensile tester. The sum of the breaking loads for these four strips is taken to represent the strength of the worn specimen, and when divided by the width of the specimen represents the strength per unit width. With such a small square of paper it is not practicable to determine the tensile strength in more than one direction of the paper. The cross direction of the paper has been tested throughout the experimental work, since this direction runs lengthwise of the paper currency. Table 1, in which the tensile strength is expressed in kilograms per centimeter width, shows the manner in which tensile strength decreases with crumpling. 
TABLE 1.-Effect of crumpling on the tensile strength of currency paper

\begin{tabular}{|c|c|}
\hline $\begin{array}{c}\text { Number of } \\
\text { times } \\
\text { crumpled }\end{array}$ & $\begin{array}{c}\text { Tensile } \\
\text { strength }\end{array}$ \\
\hline & $k g_{/} \mathrm{cm}$ \\
& 5.2 \\
8 & 3.9 \\
8 & 3.5 \\
24 & 3.3 \\
32 & $\mathbf{3 . 1}$ \\
$\mathbf{4 0}$ & $\mathbf{2 . 9}$ \\
\hline
\end{tabular}

\section{STIFFNESS}

Another property which is altered significantly in worn paper currency is stiffness. In fact, the crispness of a new note, in contrast with the limpness of a much worn one, is one of the characteristics uppermost in the mind of anyone handling paper currency. A survey made several years ago of worn paper currency in several localities indicated that the limpness, measured as the amount of bending of the paper, supported as a cantilever beam bending under its own weight, correlated fairly well with the extent of unserviceability through wear in circulation as judged by United States Treasury experts. An obvious advantage of a stiffness test is that it can be made without destroying or injuring the specimen.

Because of the shape and size of the specimen that must be tested, the conventional forms of stiffness testers are not very well suited to the purpose. A more appropriate form of apparatus was designed, and a homespun model was made with which some

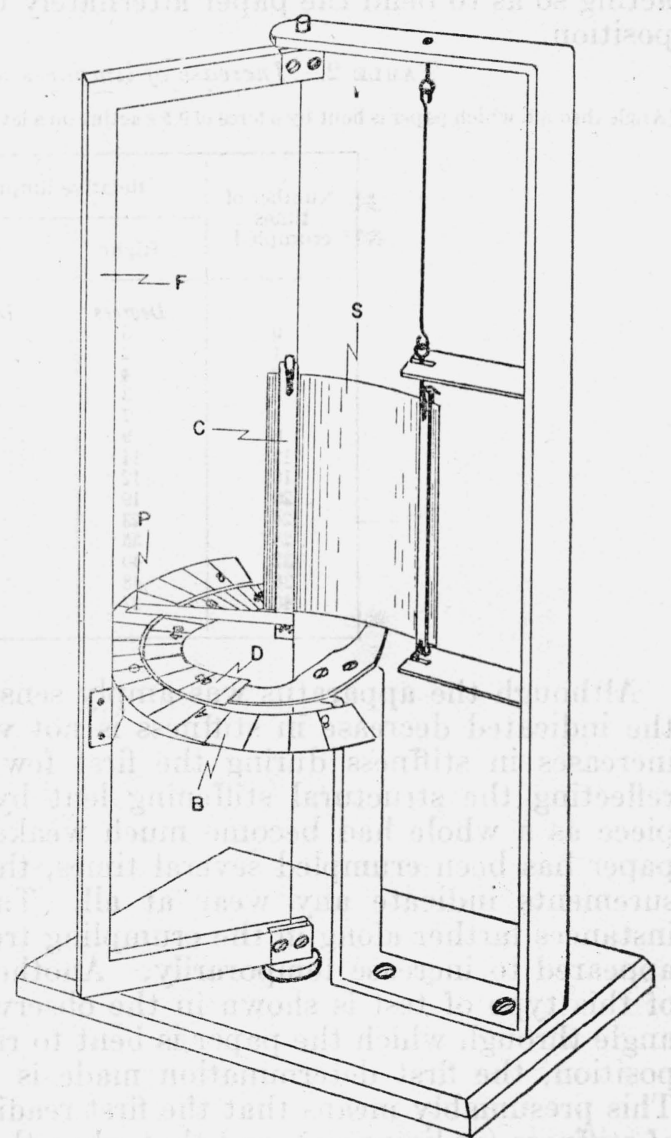

FIGURE 6.-Outline drawing showing the principle used in measuring the stiffness of crumpled paper.

experimental tests could be performed. Figure 6 illustrates the principle used in the apparatus. A clamp, $C$, is suspended between two pieces of fine piano wire held by a pivoted frame, $F$. A similar clamp 
is suspended from the fixed frame so that it can rotate freely through a small angle, or move in or out in the guides, to allow the specimen, $S$, to bend freely and naturally when clamp $C$ is rotated. Swinging the frame, $F$, to right or left about the piano wire as an axis tends to rotate the clamp, $C$, and hence to bend the specimen. A pointer, $P$, on the clamp, $C$, shows on the fixed scale, $D$, the angular deflection of the specimen. The bending moment is measured by the torsion in the piano wire, and is indicated by the position of the pointer above the scale, $B$, attached to the frame, $F$.

Tests were made at various crumpling intervals to determine the amount the paper would bend under the influence of a constant bending moment. Thus, increasing values with successive crumplings would indicate the rate of increase in limpness with repeated crumpling. Some results are shown in table 2 for a given bending moment acting so as to bend the paper alternately to right and left of the zero position.

TABLE 2.-Increase of limpness with crumpling

[Angle through which paper is bent by a force of $0.5 \mathrm{~g}$ acting on a lever arm of $4 \mathrm{~cm}$ (distance between clamps)]

\begin{tabular}{|c|c|c|}
\hline \multirow{2}{*}{$\begin{array}{c}\text { Number of } \\
\text { times } \\
\text { crumpled }\end{array}$} & \multicolumn{2}{|c|}{ Relative limpness } \\
\cline { 2 - 3 } & Right & Leit \\
\hline & Degrees & Degrees \\
0 & 3 & 3 \\
1 & 2 & 2 \\
2 & 4 & 5 \\
3 & 3 & 3 \\
4 & 7 & 3 \\
8 & 9 & 6 \\
12 & 14 & 8 \\
16 & 12 & 9 \\
20 & 19 & 15 \\
24 & 23 & 12 \\
28 & 35 & 14 \\
32 & 40 & 23 \\
36 & 48 & 23 \\
40 & 27 & 24 \\
& & \\
\hline
\end{tabular}

Although the apparatus was amply sensitive for the requirements, the indicated decrease in stiffness is not very uniform. There were increases in stiffness during the first few crumplings, undoubtedly reflecting the structural stiffening lent by the crimping before the piece as a whole had become much weakened. Not until after the paper has been crumpled several times, therefore, will stiffness measurements indicate any wear at all. Table 2 shows also several instances farther along in the crumpling treatment when the stiffness appeared to increase temporarily. Another disturbing characteristic of this type of test is shown in the observation that, in reading the angle through which the paper is bent to right and left of the neutral position, the first determination made is usually the greater angle. This presumably means that the first reading is the better indication of stiffness (or limpness), and that when the paper is bent the pattern of crumples assumes a set such as to resist bending in the opposite direction. The crumpled paper seems to behave somewhat like a piece of distorted sheet metal, resisting bending up to a certain point and then suddenly snapping over into a new position. This sudden bending was frequently observed when the bending force was increased sufficiently. 
The results of the stiffness tests indicate that, although an apparatus of this type might be developed into a very sensitive and significant measure of the stiffness of plane sheets, the measurement in this manner of the stiffiness of crumpled paper is not very reliable. Hence the apparatus has not been further developed.

Another way of getting at the stiffness was also tried. The force required to open up the crumpled wad of paper appeared to be a possible means of evaluating the relative stiffness at various stages in the crumpling process. An attempt was made to make such a measurement in place on the crumpling apparatus by attaching a small spring balance to the cord that applies tension to the crumpled specimen. It was possible to make rough measurements, which were good enough to show significant changes. Some illustrative data are shown in table 3 . This test has some promise, and may yet prove to be useful, but would require considerable refinement in order to be capable of accurate and dependable measurements.

TABLE 3.- "Uncrumpling" force at various stages in the crumpling process

\begin{tabular}{|c|c|}
\hline $\begin{array}{c}\text { Number of } \\
\text { times } \\
\text { crumpled }\end{array}$ & $\begin{array}{c}\text { Uncrumpling } \\
\text { force }\end{array}$ \\
\hline & $k \sigma$ \\
2 & 4.3 \\
6 & 3.4 \\
10 & 3.2 \\
20 & 2.2 \\
40 & 1.3 \\
\hline
\end{tabular}

\section{AIR PERMEABILITY}

\section{(a) VALUE OF AIR-PERMEABILITY MEASUREMENTS}

More sensitive than either the strength measurements or the stiffness tests, the increase in air permeability with crumpling has proved to be the most useful measure thus far found of the change resulting from the crumpling treatment. Although its relation to significant properties of the paper is somewhat indirect, the increase in air permeability appears to reflect to a very satisfactory degree the general deterioration resulting from wear. When paper is creased, the interlaced fibers are disturbed, and there is a partial breaking of the bonds which were formed on the paper machine and in subsequent processes in the manufacture of the paper. The hundreds of small creases that result from the crumpling of paper leave the specimen more open and more susceptible to the passage of air through the structure. Indirectly, this condition becomes a measure of the change in important characteristics of the paper. Loss in strength, increasing limpness, ability to absorb oil and grease, fuzziness of surface, susceptibility to catching dirt, and loss in the clarity of images printed on the paper, are all closely related to the loosening of the structure that is reflected in the increasing air permeability. An important advantage of this test is that it is nondestructive. Little or no change in the structure results from making the test. It is possible, therefore, for one to make repeated tests on the same specimen and to follow its behavior through a series of crumplings, noting the changes at all stages from new to completely unserviceable paper. The test is also very sensitive to small changes in structure. 
Some typical graphs, illustrating the consistent and regular manner in which the air permeability increases as the crumpling process goes on, are shown in figure 7 . The paper treated in this instance increased in air permeability considerably more than a hundredfold as a result of being crumpled 40 times, and the rate of increase is nearly linear. The data in figure 7 indicate that this kind of wear test is particularly suitable for appraising surface treatments and processing of currency paper. Curve $A$ represents the rate of wear of a sample of the experi-

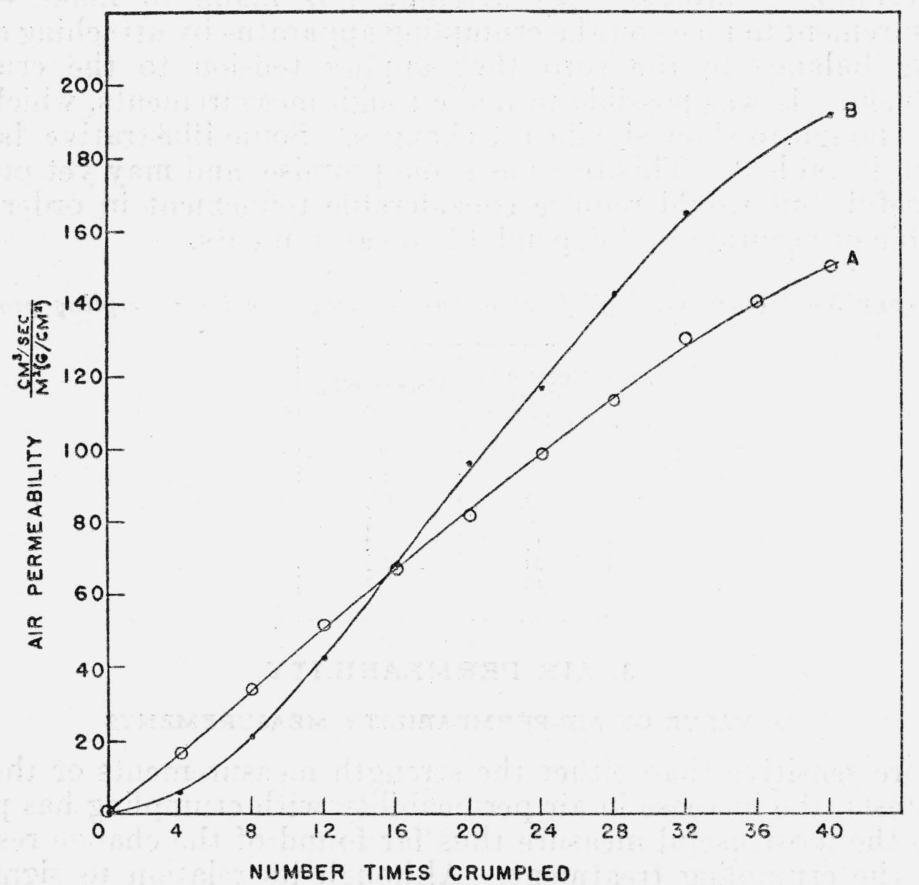

Figure 7.-Typical curves showing the manner in which air permeability increases with repeated crumpling of paper.

Air permeability is expressed as the number of cubic centimeters of air that pass per second through a square meter of the paper tested when impelled by a pressure difference of 1 gram per square centimeter (hereafter abbreviated to csm units).

mental, printed currency-type paper, while curve $B$ represents the rate of wear of the same kind of paper after it has been coated with an experimental surface sizing material intended to increase its resistance to wear. During the early stages of the crumpling treatment the performance does appear to be improved by the surface sizing, but after a short time the treated paper begins to open up more rapidly, and during the remainder of the crumpling procedure it seems to wear more rapidly than the untreated paper.

In order that figure 7 may be more intelligible, it may be noted that the condition of currency paper after 40 crumplings represents an advanced stage of wear, although probably not in the neighborhood of complete unserviceability. Air-permeability tests have been made of a few silver certificates, which the Treasury Department had withdrawn from circulation, after periods of service ranging from 4 to 17 


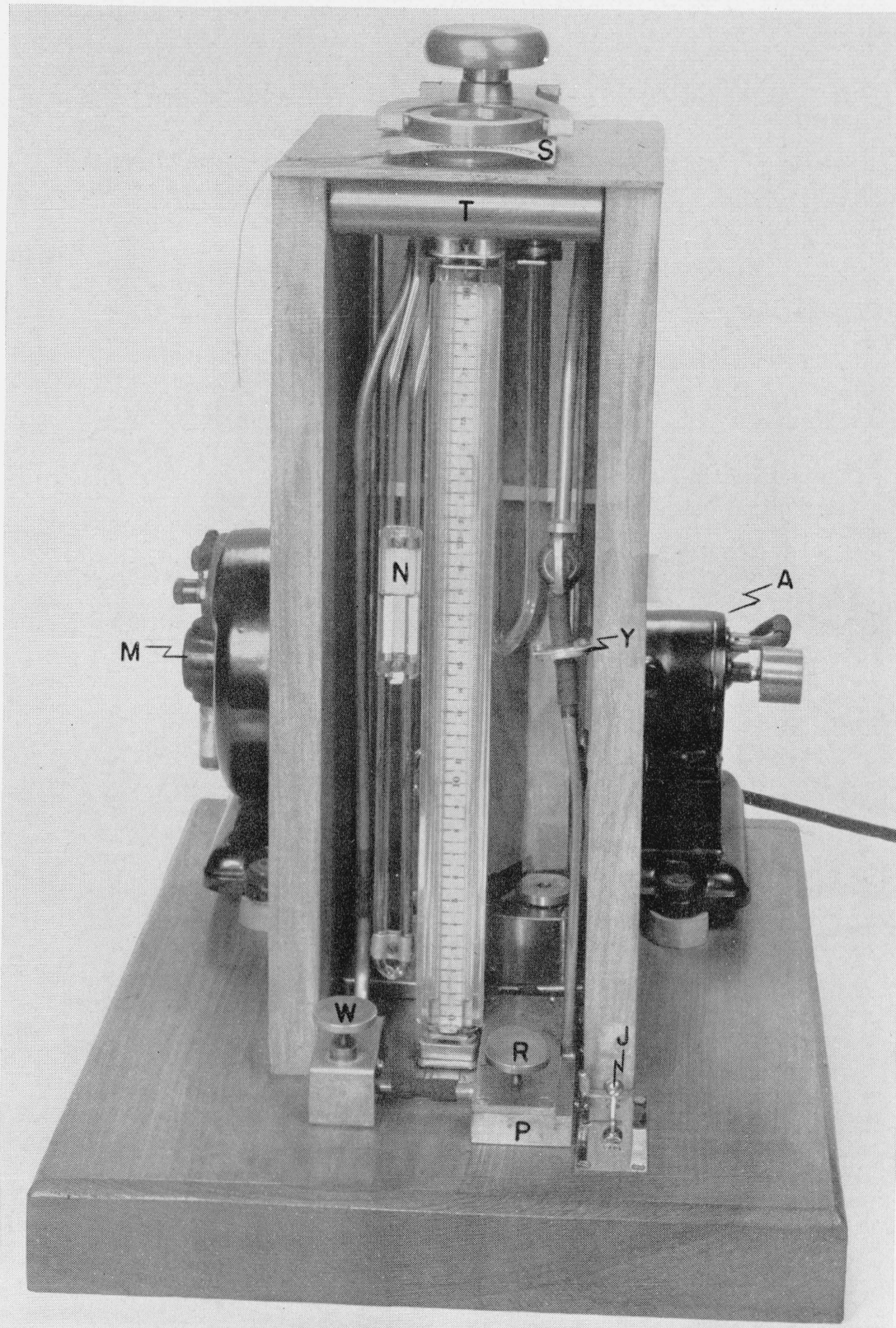

FigURE 8.-Air permeameter used as a means of measuring the break-down of the structure of paper resulting from the crumpling treatment. 
months, because they were judged to be unserviceable. The air permeability of these silver certificates ranges from 78 to 787 , with an average of about $350 \mathrm{csm}^{1}$ units.

(b) DESCRIPTION OF THE AIR-PERMEABILITY INSTRUMENT

An instrument was developed some time ago at the National Bureau of Standards, with which accurate measurements of the air permeability of paper can be made. ${ }^{2}$ It will not, however, accommodate a specimen as small as that operated on in the crumpling apparatus. Preliminary tests were made with a small, improvised cell attached

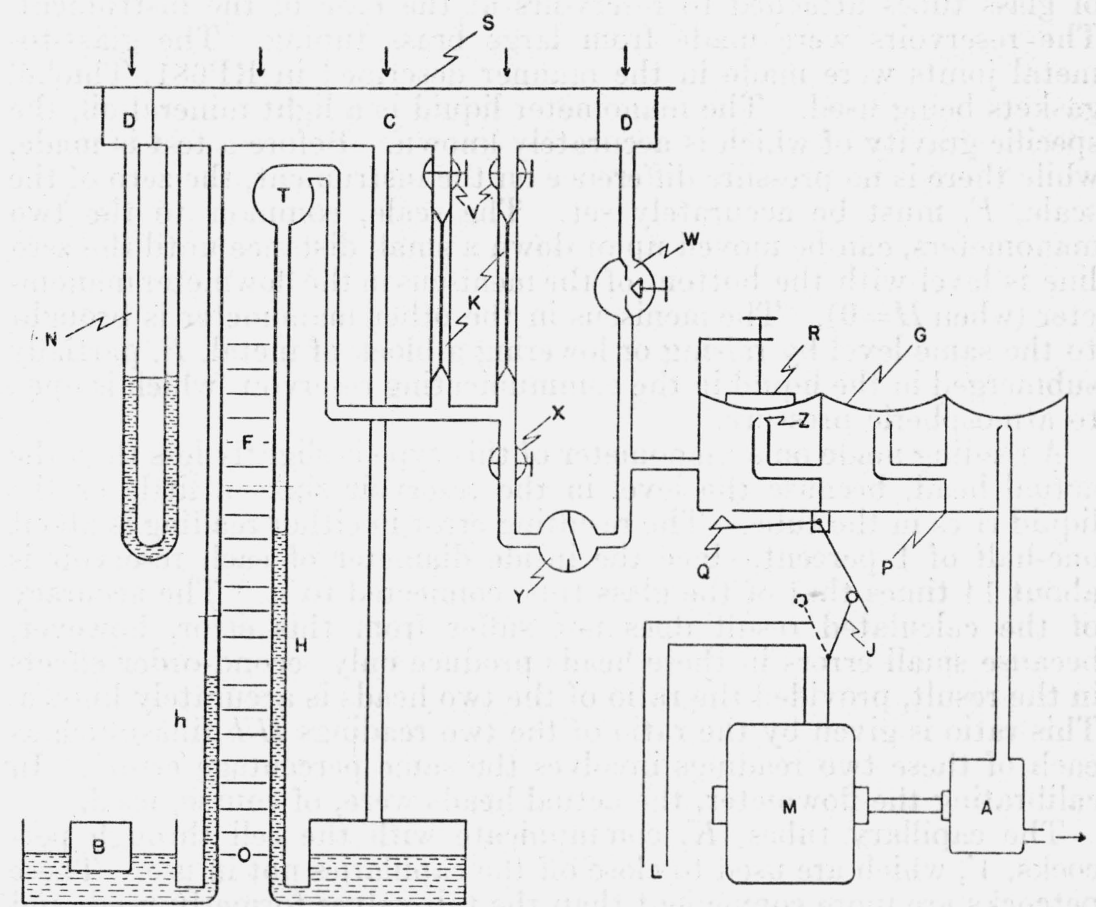

FiguRe 9.-Diagram of the air permeameter.

to this instrument. A new instrument, better suited to the requirements of the study of currency paper, has since been made. It is similar in general design to that described in RP681, but, since it differs from the older instrument in some respects, it will be described briefly. Figure 8 shows the instrument; figure 9 is a schematic diagram of it. The cell, much smaller than that in the older instrument, tests a $10-\mathrm{cm}^{2}$ area of the paper.

The specimen, $S$, is clamped over the permeability cell by means of a clamping ring (see fig. 8), the clamping pressure required being only enough to insure good contact of the specimen with the cell. Air is drawn by means of an air pump, $A$, through the specimen into the test cell, $C$ (fig. 9), and simultaneously into an annular guard cell, $D$.

\footnotetext{
1 A csm unit represents the flow of 1 cubic centimeter of air per second through 1 square meter of the material when the pressure difference across the sheet is $1 \mathrm{~g} / \mathrm{cm}^{2}$.

$2 \mathrm{~F}$. T. Carson, A sensitive instrument for measuring the air permeability of paper and other sheet materials, BS J. Research 12, 567 (1934) RP681.
} 
The function of the latter is to prevent edge leakage into the test cell. When the air flow has been so adjusted by means of the valve, $W$, that the lateral pressure betweeen the two cells (shown by the manometer, $N$ ) remains zero, no edge leakage can occur. That part of the air that flows into the test cell through the specimen passes through one of a set of capillaries, $K$, which serves as the known resistance in a capillary flowmeter. From a previous calibration the rate of flow is known for any head, $H$, indicated on the flowmeter manometer, this rate of flow being the same as that through the specimen (with pressure head $h$ ).

The manometers, except $N$, are the single-reading type, composed of glass tubes attached to reservoirs at the base of the instrument. The reservoirs were made from large brass tubing. The glass-tometal joints were made in the manner described in RP681, Thiokol gaskets being used. The manometer liquid is a light mineral oil, the specific gravity of which is accurately known. Before a test is made, while there is no pressure difference on the instrument, the zero of the scale, $F$, must be accurately set. The scale, common to the two manometers, can be moved up or down a small distance until the zero line is level with the bottom of the meniscus in the flowmeter manometer (when $H=0$ ). The meniscus in the other manometer is brought to the same level by raising or lowering a block of metal, $B$, partially submerged in the liquid in the communicating reservoir, which is open to atmospheric pressure.

A reading made on a manometer of this type is slightly less than the actual head, because the level in the reservoir sinks a little as the liquid rises in the tube. The resulting error in either reading is about one-half of 1 percent, since the inside diameter of each reservoir is about 14 times that of the glass tube connected to it. The accuracy of the calculated result does not suffer from this error, however, because small errors in these heads produce only second-order effects in the result, provided the ratio of the two heads is accurately known. This ratio is given by the ratio of the two readings $H / h$ (inasmuch as each of these two readings involves the same percentage error). In calibrating the flowmeter, the actual heads were, of course, used.

The capillary tubes, $K$, communicate with the cell through petcocks, $V$, which are used to close off the capillaries not in use. These petcocks are more convenient than the tab valves formerly used, and have given no trouble from leakage. The capillaries are of glass, about 15 inches long, bent in a $U$-shape. As a means of measuring very small rates of flow, an extremely fine capillary, made of thermometer tubing, was added to the capillary flowmeter. Its use has not proved very satisfactory, however, because of the long time required for the pressure difference across it to settle down to a steady value. There has always been considerable uncertainty in its use.

A check valve, $Y$, is placed in the line to prevent a sudden backflow of air when the motor is cut off. Without such a valve a sudden reversal of flow sometimes forces all the liquid out of the flowmeter manometer into the connecting reservoir, together with some air. On its return the liquid traps air bubbles in the manometer tube, and these are sometimes troublesome to dislodge. The check valve is simply a perforated rubber disk in contact with a perforated metal disk, the two perforations being offset. This allows free flow of air in one direction; but when the pressure difference is reversed, so as to 
press the rubber disk against the metal disk, the air leaks back slowly into the instrument.

A bypass valve, $Q$, is placed between the air pump and the flowmeter to lessen the chance of accidentally overflowing the manometer tubes or draining the manometers by suddenly throwing a large pressure difference on the instrument. When the current to the motor, $M$ (line wires indicated by $L$ ), is cut off by throwing the switch, $J$, the bypass valve is kicked open. When the switch is again closed, the valve, $Q$, remains open until it is closed by hand. While it is open, air is drawn directly from the atmosphere instead of through the instrument. As a further precaution against contaminating the capillaries with manometer liquid, an overflow reservoir, or trap, $T$, is placed between the flowmeter manometer and the capillaries.

The pulsating and somewhat irregular pressure difference produced by the air pump is made steady across the instrument by interposing a pressure regulator, $P$, a different form of which was described in RP681. The use of fixed nozzles has somewhat simplified the new regulator, which is, however, in three stages instead of two. Three wells in a block of metal contain the nozzles. A sheet of thin rubber, $G$, held by a cover plate (not shown), serves both as a gasket and as the regulating diaphragms for the three stages of the regulator. The pressure difference between the atmosphere and each of the three wells decreases progressively toward the instrument. The first two stages, next to the air pump, have permanent adjustments. In the lowest-pressure stage, next to the measuring instrument, however, the center portion of the diaphragm is moved toward or away from the nozzle, $Z$, by means of a screw-driven ring, $R$, in order to change the pressure difference across the instrument. This single adjustment has been found more convenient than the double adjustment in the older apparatus.

This small air-permeability instrument has been used to advantage not only in helping to evaluate the wear of currency paper, but has frequently made it possible to test small pieces of various other materials which could not be accommodated in the older instrument.

\section{CONCLUSION}

The instruments which have been described have proved to be very useful in evaluating the effects of various treatments of currency paper intended to make it more serviceable. Beyond this application (illustrated by fig. 7) the value of the wear test offers a fertile field for future investigation. It is felt that the crumpling treatment described is a very effective means of simulating the type of wear to which currency paper is subjected, and perhaps also of artificially wearing various papers that are subjected to creasing and much handling. However, the problem of appraising the effect of crumpling (as of wear in actual service), and assigning numerical values that represent the degree of wear, is somewhat formidable when papers are involved which differ materially in type, composition, and structure. It has already been pointed out that the interpretation of air-permeability data in terms of wear is highly inferential. Although the test has worked out well in the application which has been made, it does not follow that the wear test can be extended unmodified to all kinds of 
paper. When there are large differences in the initial air-permeability values of the papers to be compared, the significance of air permeability becomes somewhat entangled in the conflicting inferences that are possible. The development of additional criteria for the effect of the crumpling treatment seems, therefore, a likely corollary of the effort to extend the wear test to paper in general, and possibly even to the application of the test to currency-type papers that differ substantially in fiber composition or structure.

A more immediate problem is to determine the effect of common variable factors in the crumpling treatment so that the procedure may be standardized more effectively. The effects of relative humidity, rate of testing, and intensity of crumpling are outstanding examples of numerous influences about which more knowledge is required.

Work is continuing on various aspects of the standardization and the usefulness of the wear test.

Washington, March 14, 1941. 